${ }^{1}$ Departamento de Nefrología, Escuela de Medicina, Facultad de

Medicina Pontificia Universidad Católica de Chile. Santiago, Chile.

Los autores declaran no tener conflictos de interés.

Trabajo no recibió financiamiento.

Recibido el 1 de septiembre de 2020, aceptado el 7 de diciembre de 2020 .

Correspondencia a: Dr. Rodrigo Sepúlveda Palamara Departamento de Nefrología. Diagonal Paraguay 362, piso 4. Santiago, Chile. rrsepulveda@uc.cl

\section{Nueva ecuación para la estimación de la natriuresis diaria a partir de parámetros en plasma y orina aislada en población chilena}

\author{
RODRIGO A. SEPÚLVEDA ${ }^{1}$, JUAN PABLO HUIDOBRO E. ${ }^{1}$, \\ AQUILES JARA ${ }^{1}$, RODRIGO TAGLE ${ }^{1}$
}

\section{A new equation to estimate daily natriuresis from parameters in plasma and spot urine sample in the Chilean population}

Background: Excessive sodium intake is associated with increased cardiovascular morbidity and mortality. Daily sodium intake is usually inferred from sodium excretion in a 24-hour urine collection, which is cumbersome and prone to errors. Different formulas have attempted to estimate 24-hour urinary sodium from a spot urine sample. Unfortunately, their concordances are insufficient and have not been tested in our population. Aim: To develop an equation to predict 24-hour urine sodium from parameters in plasma and spot urine samples. To validate the equation and compare it with other formulas in Chilean population. Material and Methods: Analysis of 24-hour urine collections, plasma sample and spot urine sample from 174 adult outpatients ( $81 \%$ females) with an estimated glomerular filtration rate (eGFR) $\geq 60 \mathrm{~mL} /$ $\mathrm{min} / 1.73 \mathrm{~m}^{2}$. These were collected between 2015 and 2019 using standardized methods and educating patients about the correct method to collect $24 \mathrm{~h}$ urine samples. In all these patients, creatinine and electrolytes were measured in plasma and urine. A new equation was developed using a multiple linear regression model. Results: Twenty-four-hour urine sodium excretion was significantly correlated with age, weight, height, eGFR, plasma osmolarity, urine electrolytes and parameters obtained from spot urine sample, among others. The new equation had a linear correlation with 24-hour natriuresis of 0.91 and the concordance was 0.9. The predictive capacity of the new equation was better than the existing formulas. Conclusions: We developed a formula to accurately predict daily natriuresis in the Chilean population.

(Rev Med Chile 2021; 149: 178-186)

Key words: Diet Therapy; Hypertension; Natriuresis; Sodium Chloride; Urine Specimen Collection.
$\mathrm{E}$ 1 sodio $\left(\mathrm{Na}^{+}\right)$es el componente principal para la regulación del volumen extracelular (VEC) mediante la función renal. La sobrecarga crónica de $\mathrm{Na}^{+}$, además de provocar hipervolemia, aumentará el calcio en el músculo liso vascular (induciendo vasoconstricción) ${ }^{1}$ y se acumula en el intersticio, activando el sistema inmune ${ }^{2}$. Todo lo anterior contribuye al desarrollo de hipertensión arterial (HTA). Se ha demostrado, en distintas poblaciones, que la ingesta excesiva de $\mathrm{Na}^{+}$aumenta la prevalencia de $\mathrm{HTA}^{3}$. Además, se asocia a mayor mortalidad general ${ }^{4}$ y a un mayor riesgo de enfer- 
medad cardiovascular y cerebrovascular ${ }^{5,6}$. Esto ha convertido a la restricción dietética de $\mathrm{Na}^{+}$en la medida más costo-efectiva para prevenir morbilidad y mortalidad cardiovascular en la población ${ }^{7}$. La Organización Mundial de la Salud recomienda una ingesta diaria de $\mathrm{Na}^{+}<2$ g por día (o cloruro de sodio $<5 \mathrm{~g} / \mathrm{d})^{8}$. Sin embargo, este nivel de restricción se ha asociado a una mayor morbimortalidad cardiovascular por activación del sistema simpático y del eje renina-angiotensina-aldosterona. Debido a esta asociación en forma de " $U$ " entre la ingesta de $\mathrm{Na}^{+}$ y la morbimortalidad, se ha sugerido que la ingesta ideal de $\mathrm{Na}^{+}$debe estar entre 2,5 y 6 g por día ${ }^{9,10}$.

La excreción de $\mathrm{Na}^{+}$ocurre principalmente en la orina: más del $90 \%$ del $\mathrm{Na}^{+}$ingerido se elimina en la orina y solo una pequeña cantidad se excreta por las heces y el sudor ${ }^{11}$. Por esta razón, la natriuresis de $24 \mathrm{~h}\left(\mathrm{Na}_{\mathrm{u}} 24 \mathrm{~h}\right)$ se ha establecido como el patrón de referencia para evaluar la ingesta diaria de sodio ${ }^{12,13}$. Las encuestas alimentarias destinadas a predecir la ingesta de $\mathrm{Na}^{+}$tienen un rendimiento deficiente ${ }^{13,14}$. Sin embargo, la recolección de orina de 24 h suele ser engorrosa y sujeta a errores ${ }^{15}$.

Estudios anteriores han intentado predecir la $\mathrm{Na}_{\mathrm{u}} 24 \mathrm{~h}$ a partir de creatininuria y sodio en muestras de orina aislada. Sin embargo, a pesar de una buena correlación, la concordancia (capacidad de predecir exactamente la $\mathrm{Na}_{\mathrm{u}} 24 \mathrm{~h}$ ) de las diferentes fórmulas ha sido insuficiente ${ }^{16-22}$. El $\mathrm{Na}^{+}$es el principal determinante del VEC, por lo tanto, el riñón modificará la excreción de $\mathrm{Na}^{+}$de acuerdo a múltiples estímulos a lo largo del tiempo, disociando la excreción diaria de $\mathrm{Na}^{+}$con el $\mathrm{Na}^{+}$en una muestra de orina aislada. A diferencia de las proteínas y albúmina (cuya excreción diaria sí se puede estimar con análisis en orina aislada $)^{23}$, proponemos que el factor limitante es la ausencia de parámetros que nos permitan inferir el manejo renal del $\mathrm{Na}^{+}$. Afortunadamente, los índices urinarios, como la fracción excretada de $\mathrm{Na}^{+}(\mathrm{FENa})$, nos dan pistas sobre el manejo renal del sodio. Recientemente se demostró que la FENa afecta la precisión en la estimación de $\mathrm{Na}_{\mathrm{u}} 24 \mathrm{~h}^{24}$.

El objetivo principal de este estudio es desarrollar una ecuación que prediga la $\mathrm{Na}_{\mathrm{u}} 24 \mathrm{~h}$ a partir de datos antropométricos y valores de laboratorio en muestras de plasma y orina aislada. Objetivos secundarios son evaluar el rendimiento de las ecuaciones existentes para estimar la $\mathrm{Na}_{\mathrm{u}} 24 \mathrm{~h}$ en nuestra población y compararlas con la nueva fórmula.

\section{Metodología}

Analizamos retrospectivamente pacientes con examen de "Perfil Renal" realizado entre enero de 2015 y junio de 2019 en nuestro centro. El "Perfil Renal" es un examen exclusivo de nuestro centro que incluye una recolección de orina de $24 \mathrm{~h}$, muestras de plasma, orina aislada, datos demográficos y antropométricos (sexo, edad, peso, altura). Previamente, al paciente se le realizó una educación para recolectar y transportar la orina. Cuando el paciente entregaba la orina de 24 h se obtenía una muestra de sangre y orina aislada (segunda orina del día ${ }^{17}$ ). En plasma y ambas muestras de orina se midió: concentración de creatinina, sodio, potasio, cloro y osmolaridad. Basado en las mediciones anteriores, se calculó la excreción fraccionada de sodio, potasio y cloro (FENa, FEK, FECl) y el gradiente transtubular de potasio (TTKG). $\mathrm{La} \mathrm{Na} 24 \mathrm{~h}$ se obtuvo a partir de la concentración de $\mathrm{Na}^{+}$en orina $\left(\mathrm{Na}_{\mathrm{u}}\right)$ de $24 \mathrm{~h}$ y el volumen urinario.

Se incluyó a pacientes ambulatorios, adultos ( $>18$ años) y sin insuficiencia renal crónica por creatinina plasmática (filtración glomerular estimada $\left[\right.$ TFGe] $>60 \mathrm{ml} / \mathrm{min} / 1,73 \mathrm{~m}^{2}$ ). Se excluyó a sujetos con recolección urinaria de $24 \mathrm{~h}$ inadecuada según criterios de la Clínica Mayo (creatininuria 13-29 mg/kg/d en hombres y 9-26 $\mathrm{mg} / \mathrm{kg} / \mathrm{d}$ en mujeres $)^{25}$, pacientes cuya obtención de muestras de orina aislada y de $24 \mathrm{~h}$ fuera en días distintos, valores duplicados o con diferencia entre FENa en muestra de orina aislada versus $24 \mathrm{~h} \geq|30 \%|$, pacientes con hiperpotasemia $>5,5$ $\mathrm{mEq} / \mathrm{L}$ o hipopotasemia $<3,5 \mathrm{mEq} / \mathrm{L}$.

Para el cálculo del tamaño muestral, considerando una regresión lineal múltiple con $\alpha=0,05$, potencia de $80 \%$, número aproximado de 7 predictores y un tamaño del efecto del $10 \%$, se necesitó un $\mathrm{n}=140$ sujetos.

Los datos numéricos se presentan como media y desviación estándar, datos categóricos a través de frecuencias. El análisis bivariado se realizó con pruebas paramétricas: $\mathrm{t}$ de Student y correlación lineal de Pearson. Se desarrolló una nueva ecuación mediante regresión lineal múltiple en una selección aleatoria de sujetos (población de fórmula). La población restante (población de validación) se utilizó para validar la nueva ecuación y compararla con las existentes. El rendimiento de las ecuaciones se evaluó mediante análisis de asociación (correlación lineal de Pearson) y concordancia (coeficiente de 
correlación de Lin y gráficos de Bland-Altman). Todos los análisis estadísticos se realizaron utilizando el software STATA ${ }^{\circledR}$ y gráficos con GraphPad Prism ${ }^{\circledR}$. Se consideró estadísticamente significativo un valor $\mathrm{p}<0,05$.

\section{Resultados}

Se obtuvo 361 pacientes adultos, ambulatorios y sin insuficiencia renal crónica con examen de
"Perfil Renal" entre enero de 2015 y junio de 2019 (mujeres 68\%; $\mathrm{n}=245$ ). Luego de aplicar criterios de exclusión quedó un total de 174 sujetos. De estos, fueron seleccionados aleatoriamente 144 sujetos para constituir la población con la cual se desarrolló la fórmula. Los sujetos restantes se utilizaron como población de validación para evaluar y comparar las fórmulas. La Tabla 1 resume las características de cada población (total, fórmula y validación).

Tabla 1. Características de la población total, de fórmula y validación

\begin{tabular}{|c|c|c|c|}
\hline Variable & $\begin{array}{l}\text { Población total* } \\
\quad(n=174)\end{array}$ & $\begin{array}{l}\text { Población fórmula* } \\
\qquad(n=144)\end{array}$ & $\begin{array}{l}\text { Población validación* } \\
\qquad(n=30)\end{array}$ \\
\hline Edad (años) & $49,2 \quad( \pm 15,8)$ & $49,0 \quad( \pm 16,2)$ & $50,4 \quad( \pm 13,9)$ \\
\hline Sexo femenino (\%) & $140 \quad(80,5 \%)$ & $116 \quad(80,6 \%)$ & $(80 \%)$ \\
\hline Talla (m) & $1.619 \quad( \pm 0,1)$ & $1.619 \quad( \pm 0,1)$ & $1.621 \quad( \pm 0,1)$ \\
\hline Peso (kg) & $67,0 \quad( \pm 13,2)$ & $66,8 \quad( \pm 13,1)$ & $68,2 \quad( \pm 13,5)$ \\
\hline $\mathrm{IMC}\left(\mathrm{kg} / \mathrm{m}^{2}\right)$ & $25,5 \quad( \pm 4,4)$ & $25,5 \quad( \pm 4,5)$ & $25,9 \quad( \pm 3,8)$ \\
\hline SCT Dubois $\left(m^{2}\right)$ & $1,71 \quad( \pm 0,2)$ & $1,71 \quad( \pm 0,2)$ & $1,72 \quad( \pm 0,2)$ \\
\hline TFGe (CKD-EPI) (mL/min/1.73 m²) & $91,3 \quad( \pm 17,9)$ & $91,7 \quad( \pm 18,1)$ & $89,9 \quad( \pm 17,5)$ \\
\hline $\begin{array}{l}\text { Mediciones en plasma } \\
\text { - } \mathrm{Na}^{+}(\mathrm{mEq} / \mathrm{L}) \\
\text { - } \mathrm{K}^{+}(\mathrm{mEq} / \mathrm{L}) \\
-\mathrm{Cl}(\mathrm{mEq} / \mathrm{L}) \\
\text { - }\end{array}$ & $\begin{array}{rr}139,9 & ( \pm 3,1) \\
4,6 & ( \pm 0,4) \\
102,0 & ( \pm 3,0) \\
297,2 & ( \pm 8,7)\end{array}$ & $\begin{array}{rr}139,9 & ( \pm 3,1) \\
4,4 & ( \pm 0,4) \\
102,0 & ( \pm 2,9) \\
297,1 & ( \pm 8,3)\end{array}$ & $\begin{array}{rr}139,8 & ( \pm 3,5) \\
4,6 & ( \pm 0,3) \\
101,4 & ( \pm 3,4) \\
297,3 & ( \pm 10,5)\end{array}$ \\
\hline $\begin{array}{l}\text { Orina de } 24 \mathrm{~h} \\
\text { - } \operatorname{Diuresis}^{(\mathrm{L})} \\
\text { - } \mathrm{Cr}_{u}(\mathrm{mg} / \mathrm{d}) \\
\text { - } \mathrm{Na}_{u}(\mathrm{mEq} / \mathrm{d}) \\
\text { - } \mathrm{K}_{u}(\mathrm{mEq} / \mathrm{d}) \\
-\mathrm{Cl}_{u}(\mathrm{mEq} / \mathrm{d}) \\
\text { - } \operatorname{Osm}_{\mathrm{u}}(\mathrm{mOsm} / \mathrm{L})\end{array}$ & $\begin{array}{r}1,815( \pm 1,112) \\
1.101,1( \pm 319,1) \\
115,6( \pm 54,3) \\
59,2( \pm 23,3) \\
106,0( \pm 51,1) \\
447,3( \pm 197,7)\end{array}$ & $\begin{array}{r}1,860( \pm 1,171) \\
1.097,3( \pm 315,0) \\
116,0( \pm 55,4) \\
59,2( \pm 23,4) \\
106,8( \pm 51,2) \\
437,6( \pm 197,7)\end{array}$ & $\begin{array}{r}1,599( \pm 752,3) \\
1.119,2( \pm 343,3) \\
113,8( \pm 49,9) \\
59,1 \quad( \pm 23,6) \\
102,0( \pm 51,2) \\
494,0( \pm 194,0)\end{array}$ \\
\hline 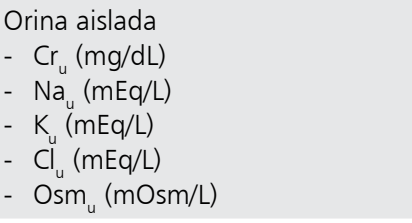 & $\begin{array}{r}125,1 \quad( \pm 76,9) \\
95,6 \quad( \pm 45,3) \\
64,6 \quad( \pm 35,4) \\
110,6 \quad( \pm 57,3) \\
590,2( \pm 219,4)\end{array}$ & $\begin{array}{rr}123,9 & ( \pm 78,5) \\
94,6 & ( \pm 44,9) \\
63,4 & ( \pm 36,3) \\
109,8 & ( \pm 55,5) \\
579,3 & ( \pm 218,0)\end{array}$ & $\begin{array}{rr}130,7 & ( \pm 69,6) \\
100,4 & ( \pm 47,6) \\
70,1 & ( \pm 31,6) \\
114,5 & ( \pm 66,2) \\
642,7 & ( \pm 222,1)\end{array}$ \\
\hline 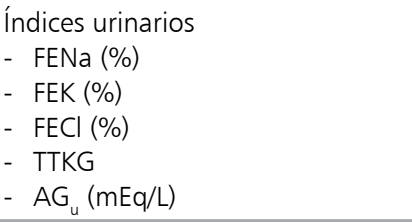 & $\begin{array}{cc}0,58 & ( \pm 0,3) \\
10,8 & ( \pm 4,9) \\
0,87 & ( \pm 0,5) \\
7,37 & ( \pm 2,6) \\
49,59 & ( \pm 33,5)\end{array}$ & $\begin{array}{cc}0,59 & ( \pm 0,3) \\
10,7 & ( \pm 4,9) \\
0,87 & ( \pm 0,4) \\
7,30 & ( \pm 2,7) \\
48,26 & ( \pm 30,4)\end{array}$ & $\begin{array}{rr}0,55 & ( \pm 0,3) \\
11,65 & ( \pm 4,9) \\
0,85 & ( \pm 0,5) \\
7,69 & ( \pm 2,4) \\
55,96 & ( \pm 45,8)\end{array}$ \\
\hline
\end{tabular}

* Sin diferencia estadística para cada variable entre grupos. Abreviaciones: AG: Anion Gap urinario; CKD-EPI: Chronic Kidney Disease Epidemiology Collaboration; $\mathrm{Cl}$ : cloro; $\mathrm{Cl}_{\mathrm{u}}$ : cloro urinario; $\mathrm{Cr}_{\mathrm{u}}$ : creatinina urinaria; $\mathrm{FECl}$ : fracción excretada de cloro; FEK: fracción excretada de potasio; FENa: fracción excretada de sodio; IMC: índice de masa corporal; $\mathrm{K}^{+}$: potasio; $\mathrm{K}_{u}$ : potasio urinario; $\mathrm{Na}^{+}$: sodio; $\mathrm{Na}_{u}$ : sodio urinario; Osm $\mathrm{u}_{\mathrm{u}}$ osmolaridad urinaria; SCT: superficie corporal total; TFGe: tasa de filtración glomerular estimada; TTKG: gradiente transtubular de potasio. 
En la población total se realizó análisis bivariado entre la $\mathrm{Na}_{\mathrm{u}} 24 \mathrm{~h}$ y datos antropométricos, valores obtenidos en plasma y mediciones en muestra de orina aislada. El sexo femenino se asoció significativamente con una menor $\mathrm{Na}_{\mathrm{u}} 24 \mathrm{~h}(\mathrm{p}=0,038)$. Las variables que tuvieron una correlación lineal positiva significativa con $\mathrm{Na}_{\mathrm{u}} 24 \mathrm{~h}$ fueron: peso, altura, superficie corporal total, TFGe, $\mathrm{Na}_{\mathrm{u}}, \mathrm{Cl}_{\mathrm{u}}$,

Tabla 2. Correlación lineal entre la natriuresis de $\mathbf{2 4}$ h y variables cuantitativas antropométricas, plasmáticas y de orina aislada

\begin{tabular}{|c|c|c|}
\hline Variable & $\begin{array}{c}\text { Coeficiente } \\
\mathbf{r}\end{array}$ & $\begin{array}{c}\text { Valor } \\
\text { p }\end{array}$ \\
\hline Edad (años)* & $-0,20$ & 0,009 \\
\hline Peso $(\mathrm{kg})^{*}$ & 0,17 & 0,022 \\
\hline Talla $(\mathrm{cm})^{*}$ & 0,20 & 0,007 \\
\hline SCT Dubois $\left(m^{2}\right)^{*}$ & 0,21 & 0,005 \\
\hline IMC & 0,07 & 0,391 \\
\hline Creatinina plasmática $(\mathrm{mg} / \mathrm{dL})$ & 0,02 & 0,835 \\
\hline TFGe $\left(\mathrm{mL} / \mathrm{min} / 1,73 \mathrm{~m}^{2}\right)^{*}$ & 0,02 & 0,008 \\
\hline $\mathrm{Na}^{+}$plasmático $(\mathrm{mEq} / \mathrm{L})$ & $-0,12$ & 0,102 \\
\hline $\mathrm{K}^{+}$plasmático (mEq/L) & 0,03 & 0,693 \\
\hline $\mathrm{Cl}^{-}$plasmático (mEq/L) & $-0,11$ & 0,145 \\
\hline $\begin{array}{l}\text { Osmolaridad plasmática } \\
(\mathrm{mOsm} / \mathrm{L})^{*}\end{array}$ & $-0,22$ & 0,003 \\
\hline $\mathrm{Cr}_{\mathrm{u}}(\mathrm{mg} / \mathrm{dL})^{*}$ & $-0,53$ & 0,000 \\
\hline $\mathrm{Na}_{\mathrm{u}}(\mathrm{mEq} / \mathrm{L})^{*}$ & 0,34 & 0,000 \\
\hline $\mathrm{K}_{\mathrm{u}}(\mathrm{mEq} / \mathrm{L})^{*}$ & $-0,29$ & 0,000 \\
\hline $\mathrm{Cl}_{\mathrm{u}}(\mathrm{mEq} / \mathrm{L})^{*}$ & 0,21 & 0,006 \\
\hline $\mathrm{Osm}_{\mathrm{u}}(\mathrm{mOsm} / \mathrm{L})^{*}$ & $-0,24$ & 0,002 \\
\hline$A G_{u}(m E q / L)^{*}$ & $-0,20$ & 0,007 \\
\hline $\mathrm{FENa}(\%)^{*}$ & 0,82 & 0,000 \\
\hline $\operatorname{FEK}(\%)^{*}$ & 0,30 & 0,000 \\
\hline $\mathrm{FECl}(\%)^{*}$ & 0,76 & 0,000 \\
\hline TTKG* & $-0,27$ & 0,000 \\
\hline
\end{tabular}

*Correlación lineal estadísticamente significativa, Abreviaciones: $\mathrm{AG}_{\mathrm{u}}$ : Anion Gap urinario; $\mathrm{Cl}$ : cloro; $\mathrm{Cl}_{\mathrm{u}}$ : cloro urinario; $\mathrm{Cr}_{u}$ : creatinina urinaria; $\mathrm{FECl}$ : fracción excretada de cloro; FEK: fracción excretada de potasio; FENa: fracción excretada de sodio; IMC: índice de masa corporal; $\mathrm{K}^{+}$: potasio; $\mathrm{K}_{4}$ : potasio urinario; $\mathrm{Na}^{+}$, sodio; $\mathrm{Na}_{\mathrm{u}^{\prime}}$ sodio urinario; $\mathrm{Osm}_{\mathrm{u}^{\prime}}$ osmolaridad urinaria; SCT: superficie corporal total; TFGe: tasa de filtración glomerular estimada; TTKG: gradiente transtubular de potasio,
FENa, FEK, FECl y TTKG. Las variables que tuvieron correlación lineal negativa significativa con el $\mathrm{Na}_{\mathrm{u}}$ 24h fueron: edad, osmolaridad plasmática, creatinina urinaria, $\mathrm{K}_{\mathrm{u}}$, osmolaridad urinaria y Anion Gap urinario $\left(\mathrm{AG}_{\mathrm{u}}\right)$. La Tabla 2 resume las asociaciones entre la $\mathrm{Na}_{\mathrm{u}} 24 \mathrm{~h}$ y variables cuantitativas con su respectiva correlación lineal y valor $\mathrm{p}$.

En la población de fórmula se desarrolló un modelo predictivo de la $\mathrm{Na}_{\mathrm{u}} 24 \mathrm{~h}$ priorizando la bondad de ajuste. Luego de realizar una regresión lineal múltiple, las variables incluidas en el modelo predictivo fueron: edad, sexo, peso, talla, TFGe, $\mathrm{K}_{\mathrm{u}}, \mathrm{AG}_{\mathrm{u}}$, FENa y FECl. Sus componentes se resumen en la Tabla 3. La Figura 1 muestra la fórmula desarrollada. El coeficiente de determinación ajustado de la fórmula fue $80 \%$.

La correlación lineal entre la $\mathrm{Na} 24$ h en la población de validación y la $\mathrm{Na}_{4} 24$ h estimada (eNa $24 \mathrm{~h})$ según la fórmula desarrollada fue $\mathrm{r}=0,91$ (Figura 2). Se analizaron otras fórmulas para $\mathrm{eNa}_{\mathrm{u}} 24 \mathrm{~h}$ disponibles en la literatura con la población de validación: Tanaka ${ }^{16}$, Kawasaki ${ }^{17}$, Toft $^{18}$, INTERSALT ${ }^{19}$, Nerbass ${ }^{20}$ y las 3 fórmulas de $\mathrm{Rhee}^{21}$. Todas presentaron asociación lineal positiva significativa (Tabla 4 ).

\section{Tabla 3. Variables seleccionadas para fórmula predictiva de natriuresis en $\mathbf{2 4}$ h mediante modelamiento lineal múltiple}

\begin{tabular}{|lccc|}
\hline Variable & $\begin{array}{c}\text { Coeficiente } \\
\boldsymbol{\beta}\end{array}$ & $\begin{array}{c}\text { Valor } \\
\mathbf{p}\end{array}$ & $\begin{array}{c}\text { IC-95\% } \\
\text { de } \boldsymbol{\beta}\end{array}$ \\
\hline Edad (años) & $-0,19$ & 0,29 & $-0,55-0,17$ \\
\hline Sexo femenino & $-10,82$ & 0,12 & $-24,43-2,78$ \\
\hline Peso (kg) & 0,26 & 0,18 & $-0,12-0,65$ \\
\hline Talla (cm) & 0,29 & 0,41 & $-0,40-0,98$ \\
\hline $\begin{array}{l}\text { TFGe } \\
\text { (mL/min/1,73 m²) }\end{array}$ & 0,90 & 0,00 & $0,58-1,21$ \\
\hline $\mathrm{K}_{\mathrm{u}}(\mathrm{mEq} / \mathrm{L})$ & 0,28 & 0,07 & $-0,02-0,57$ \\
\hline $\mathrm{AG}(\mathrm{mEq} / \mathrm{L})$ & $-0,13$ & 0,35 & $-0,42-0,15$ \\
\hline $\mathrm{FENa}(\%)$ & 196 & 0,00 & $132,43-259,64$ \\
\hline FECl $(\%)$ & $-34,10$ & 0,13 & $-77,69-9,52$ \\
\hline Constante & $-109,45$ & 0,09 & $-237,54-18,64$ \\
\hline
\end{tabular}

Abreviaciones: $\mathrm{AG}_{4}$ : Anion Gap urinario; FECl: fracción excretada de cloro; FENa: fracción excretada de sodio; IC: intervalo de confianza; $K_{u}$ : potasio urinario; TFGe: tasa de filtración glomerular estimada. 


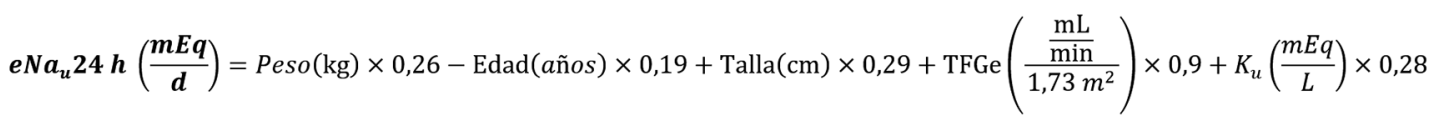

$$
\begin{aligned}
& -A G_{u}\left(\frac{m E q}{L}\right) \times 0,13+F E N a(\%) \times 196-F E C l(\%) \times 34-109-11 \text { si mujer }
\end{aligned}
$$

Figura 1. Fórmula para estimar natriuresis de $24 \mathrm{~h}$. $A \mathrm{G}$ : Anion Gap urinario; eNa $24 \mathrm{~h}$ : natriuresis de $24 \mathrm{~h}$ estimado; $\mathrm{FECl}$ : fracción excretada de cloro; FENa: fracción excretada de sodio; $K_{u}$ : potasio urinario; TFGe: tasa de filtración glomerular estimada.

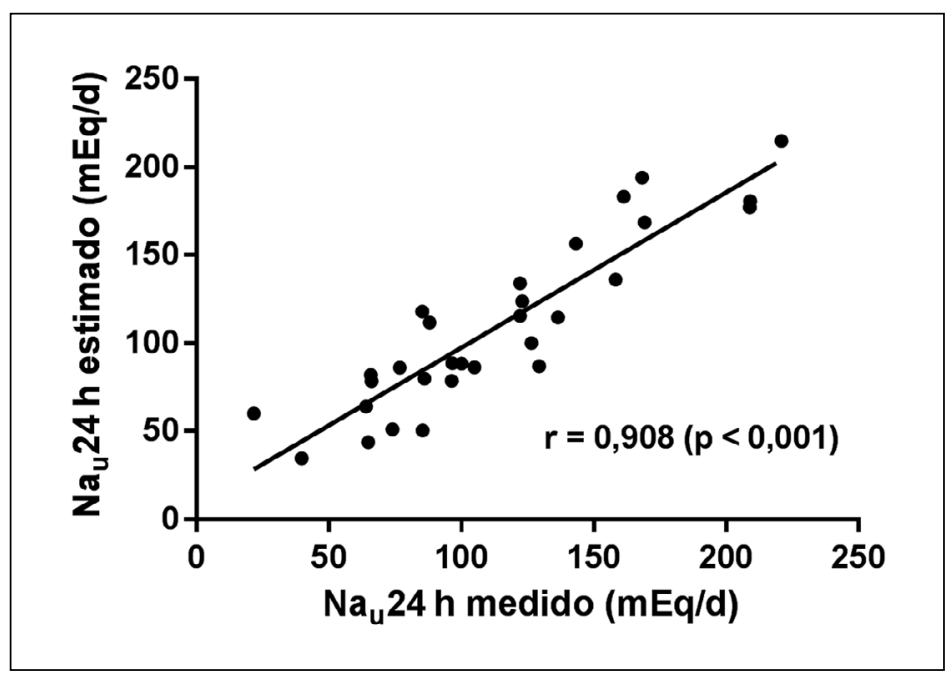

Figura 2. Correlación lineal entre natriuresis de 24 h ( $\mathrm{Na}_{\mathrm{u}} 24 \mathrm{~h}$ ) medida y estimada en población de validación.

Tabla 4. Correlación, precisión y concordancia entre la natriuresis de $\mathbf{2 4}$ h medida y fórmulas predictivas

\begin{tabular}{|c|c|c|c|}
\hline Fórmula & $\begin{array}{l}\text { Coeficiente de } \\
\text { correlación lineal } \\
\text { (valor p) }\end{array}$ & $\begin{array}{l}\text { Coeficiente de } \\
\text { concordancia de Lin } \\
\text { (IC-95\%) }\end{array}$ & $\begin{array}{l}\text { Promedio de la di- } \\
\text { ferencia de medidas } \\
\text { (DE) }\end{array}$ \\
\hline eNa 24 h por fórmula desarrollada & $0,91(0,00)$ & $0,90(0,84-0,97)$ & $4,14(21,18)$ \\
\hline eNa 24 h Tanaka ${ }^{16}$ & $0,89(0,00)$ & $0,72(0,59-0,86)$ & $-23,69(24,77)$ \\
\hline eNa 24 h Kawasaki ${ }^{17}$ & $0,91(0,00)$ & $0,60(0,45-0,75)$ & $-51,42(22,78)$ \\
\hline $\mathrm{eNa}_{\mathrm{u}} 24 \mathrm{~h} \mathrm{Toft}^{18}$ & $0,67(0,00)$ & $0,53(0,32-0,73)$ & $-25,60(36,91)$ \\
\hline eNa 24 h INTERSALT ${ }^{19}$ & $0,59(0,00)$ & $0,55(0,32-0,79)$ & $-3,46(40,50)$ \\
\hline $\mathrm{eNa}_{\mathrm{u}} 24$ h Nerbass ${ }^{20}$ & $0,49(0,01)$ & $0,46(0,18-0,73)$ & $9,63(45,58)$ \\
\hline $\mathrm{eNa}_{\mathrm{u}} 24 \mathrm{~h}$ Rhee lineal ${ }^{21}$ & $0,90(0,00)$ & $0,79(0,67-0,90)$ & $-21,70(22,02)$ \\
\hline eNa 24 h Rhee cuadrática ${ }^{21}$ & $0,90(0,00)$ & $0,78(0,66-0,90)$ & $-21,99(22,34)$ \\
\hline eNa 24 h Rhee cúbica ${ }^{21}$ & $0,91(0,00)$ & $0,79(0,68-0,90)$ & $-21,23(21,89)$ \\
\hline
\end{tabular}

Abreviaciones: DE: desviación estándar; IC: intervalo de confianza; eNa 24 h: natriuresis de 24 h estimada. 


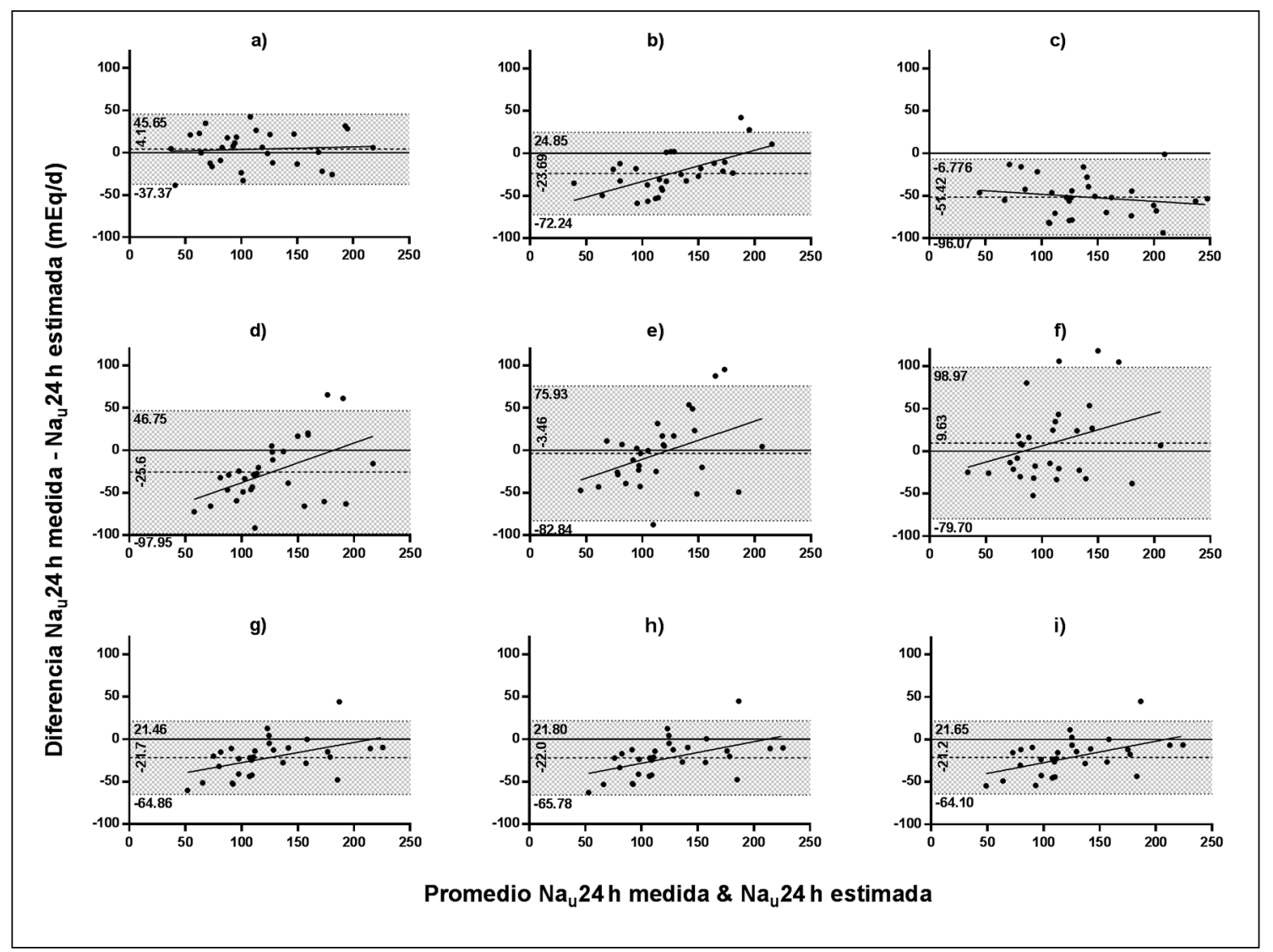

Figura 3. Gráficos de Bland-Altman entre natriuresis de $24 \mathrm{~h}$ medida y estimada por fórmulas en población de validación. a) Nuestra fórmula, b) Tanaka $^{16}$, c) Kawasaki ${ }^{17}$, d) Toft ${ }^{18}$, e) INTERSALT ${ }^{19}$, f) Nerbass ${ }^{20}$, g) Rhee lineal ${ }^{21}$, h) Rhee cuadrática ${ }^{21}$, i) Rhee cúbica ${ }^{21} \cdot \mathrm{Na}_{\mathrm{u}} 24 \mathrm{~h}$ : natriuresis de $24 \mathrm{~h}$.

El coeficiente de correlación de concordancia de Lin para la fórmula desarrollada fue $\rho=0,90$. Para las otras fórmulas (Tanaka, Kawasaki, Toft, INTERSALT, Nerbass y las 3 fórmulas de Rhee); fueron respectivamente: $\rho=0,72 ; \rho=0,60$; $\rho=0,53 ; \rho=0,55, \rho=0,46 ; \rho=0,79 ; \rho=0,78$ y $\rho=0,79$. La Tabla 4 muestra las estimaciones de concordancia y la Figura 3 los gráficos de Bland-Altman por fórmula.

\section{Discusión}

Hasta donde sabemos, la fórmula desarrollada en este trabajo es la primera en incluir parámetros plasmáticos para estimar la $\mathrm{Na} 24 \mathrm{~h}$. Las ecuaciones existentes se basan en la hipótesis de que la relación entre la "concentración urinaria aleatoria" de $\mathrm{Na}_{u}$ y $\mathrm{Cr}_{u}$ en orina aislada es proporcional a la relación entre $\mathrm{Na}_{u}$ y $\mathrm{Cr}_{u}$ en orina de 24 h. Esta afirmación puede ser correcta para moléculas que solo se filtran o filtran y tienen reabsorción tubular constante (como las proteínas). Sin embargo, como el $\mathrm{Na}^{+}$es el principal elemento de control del VEC, su excreción (medida como concentración) tiene grandes variaciones en el tiempo y se disocia de la normalización por el volumen urinario que aporta la creatinina. Esto último puede ser el factor limitante de las fórmulas predictivas de $\mathrm{Na}_{\mathrm{u}} 24 \mathrm{~h}$.

Hay muchas vías biológicas que afectan la excreción de sodio. Como no es posible medir cada una de estas vías, parece lógico cuantificar el efector final común: la fracción excretada de electrolitos urinarios. Con el uso de índices 
urinarios (como FENa y FEK) es posible inferir el comportamiento renal debido a la suma de sistemas biológicos que afectan la excreción de sodio.

En nuestra población, muchos sujetos no fueron incluidos debido a una recolección de orina inadecuada. Esto refuerza la necesidad de contar con fórmulas predictivas de parámetros urinarios de $24 \mathrm{~h}$. No se incluyeron pacientes hospitalizados, por la variabilidad que pudieran tener en su función renal así como los sujetos con enfermedad renal crónica.

Una limitación del estudio es la ausencia de información sobre los antecedentes médicos de los pacientes o el uso de medicamentos. Por ejemplo, el uso de diuréticos podría afectar la excreción de $\mathrm{Na}^{+}$y disociarlo del consumo dietético actual. Sin embargo, estas situaciones también afectan el valor de la $\mathrm{Na}_{\mathrm{u}} 24$ h como reflejo del consumo real de sodio, lo que determina que no existe un patrón de referencia para estimar la ingesta de $\mathrm{Na}^{+}$en estas circunstancias. Como nuestro objetivo era estimar $\mathrm{Na}_{\mathrm{u}} 24 \mathrm{~h}$, la fórmula no se ve afectada por los factores mencionados.

La $\mathrm{Na}_{\mathrm{u}} 24 \mathrm{~h}$ promedio fue 115,6 mEq/d (6,8 g de $\mathrm{Na}^{+}$), un valor alto considerando las recomendaciones internacionales. La FENa promedio fue $0,58 \%$, probablemente debido al estado de ayuno realizado antes de la obtención de los exámenes. La mayoría de la población estudiada fueron mujeres.

La selección aleatoria de la población de validación fue adecuada, como lo demuestra la similitud de las 3 poblaciones (total, fórmula y validación). La fórmula de población $(\mathrm{n}=144)$ satisface el tamaño de muestra estimado. El tamaño de la población de validación $(\mathrm{n}=30)$ por el teorema del límite central permite pruebas paramétricas.

La $\mathrm{Na}_{\mathrm{u}} 24 \mathrm{~h}$ se asoció con el tamaño de los sujetos (altura y superficie corporal total), pero no con obesidad (índice de masa corporal). Como era de esperar, las concentraciones urinarias de solutos $\left(\mathrm{Cr}_{\mathrm{u}}, \mathrm{Osm}_{\mathrm{u}}\right)$ tuvieron una correlación lineal negativa, ya que una mayor natriuresis arrastra más agua diluyendo los demás componentes. Es interesante destacar el comportamiento del $\mathrm{K}^{+}$: tanto la concentración de $\mathrm{K}^{+}$urinario como la TTKG en orina aislada tuvieron correlación lineal negativa. El TTKG indica la acción del eje renina-angiotensina-aldosterona, por lo tanto, una menor excreción urinaria de $\mathrm{Na}^{+}$se correlaciona con una mayor excreción de $\mathrm{K}^{+}$urinario. Los parámetros plasmáticos no mostraron una asociación lineal con el $\mathrm{Na}_{\mathrm{u}} 24 \mathrm{~h}$, pero sí los índices urinarios. Esto refuerza la idea de utilizar índices urinarios para estimar los parámetros urinarios de $24 \mathrm{~h}$.

La fórmula desarrollada, junto a las de Rhee, Tanaka y Kawasaki, tuvieron muy buenas correlaciones lineales en la población de validación. Sin embargo, incluso si una asociación es perfecta y significativa, no permite la predicción de la $\mathrm{Na}_{\mathrm{u}} 24 \mathrm{~h}$ real. Por esta razón, utilizamos la evaluación de la concordancia utilizando el coeficiente de correlación de Lin. En los análisis de concordancia, la fórmula desarrollada fue superior a las ecuaciones existentes. El coeficiente de correlación de Lin superó el 90\%, siendo el único que logró este excelente grado de precisión. Cabe señalar que las fórmulas de Rhee, Tanaka, Kawasaki y Toft presentaron coeficientes de concordancia aceptables, sin embargo, tuvieron una diferencia de medidas promedio muy negativa. Eso significa que estas fórmulas sobrestiman sistemáticamente el Na $24 \mathrm{~h}$. Las ecuaciones de INTERSALT y Nerbass tienen un amplio rango de dispersión.

Los gráficos de Bland-Altman muestran el comportamiento de cada método de estimación, destacando que nuestra fórmula es la más concordante con la $\mathrm{Na}_{\mathrm{u}} 24 \mathrm{~h}$. La fórmula de Kawasaki también presenta una concordancia aceptable, sin embargo, probablemente requiera un factor de corrección, al menos en la población estudiada, ya que sobreestima sistemáticamente la $\mathrm{Na}_{\mathrm{u}} 24 \mathrm{~h}$. Las ecuaciones de Tanaka, Toft, INTERSALT y Nerbass sobrestimaron sistemáticamente los valores bajos de $\mathrm{Na}_{\mathrm{u}} 24 \mathrm{~h}$ medidas y subestimaron los valores altos de $\mathrm{Na}_{\mathrm{u}} 24 \mathrm{~h}$.

Las limitaciones de nuestro estudio son su diseño retrospectivo, realización en un solo centro con población exclusivamente chilena y un tamaño muestral moderado. Todo lo anterior disminuye la validez externa. Sin embargo, no existe otro estudio con una fórmula predictiva para $\mathrm{Na}_{\mathrm{u}} 24 \mathrm{~h}$ con una concordancia tan alta como la nuestra. Por lo tanto, creemos que agregar índices urinarios y parámetros plasmáticos a las ecuaciones predictivas permite optimizar la capacidad predictiva de las ecuaciones. Un estudio multicéntrico prospectivo con $\mathrm{n}$ mayor podría encontrar fórmulas predictivas aun más precisas.

Podemos recomendar el uso de nuestra ecuación para estimar la $\mathrm{Na}_{\mathrm{u}} 24 \mathrm{~h}$. Si no es posible contar con muestras de plasma ni índices urinarios, 
se podría utilizar las fórmulas de Rhee, ya que, en nuestra población, tiene una menor capacidad predictiva pero superior a las otras fórmulas. Así, conociendo el consumo diario de $\mathrm{Na}^{+}$se podría tomar medidas preventivas poblacionales para reducir la morbimortalidad cardiovascular.

\section{Referencias}

1. Iwamoto T. Vascular $\mathrm{Na}^{+} / \mathrm{Ca}^{2+}$ exchanger: implications for the pathogenesis and therapy of salt-dependent hypertension. Am J Physiol Regul Integr Comp Physiol. 2006; 290 (3): R536-R545.

2. Wiig H, Luft FC, Titze JM. The interstitium conducts extrarenal storage of sodium and represents a third compartment essential for extracellular volume and blood pressure homeostasis. Acta Physiol (Oxf). 2018; 222 (3): 10. 1111/apha.13006.

3. Dahl LK. Possible role of salt intake in the development of essential hypertension. 1960. Int J Epidemiol. 2005;34 (5): 967-78.

4. Cook NR, Appel LJ, Whelton PK. Sodium Intake and All-Cause Mortality Over 20 Years in the Trials of Hypertension Prevention. J Am Coll Cardiol. 2016; 68 (15): 1609-17.

5. Cook NR, Cutler JA, Obarzanek E, Buring JE, Rexrode KM, Kumanyika SK, et al. Long term effects of dietary sodium reduction on cardiovascular disease outcomes: observational follow-up of the trials of hypertension prevention (TOHP). BMJ. 2007; 334 (7599): 885-8.

6. Strazzullo P, D’Elia L, Kandala NB, Cappuccio FP. Salt intake, stroke, and cardiovascular disease: meta-analysis of prospective studies. BMJ. 2009; 339: b4567.

7. Asaria P, Chisholm D, Mathers C, Ezzati M, Beaglehole R. Chronic disease prevention: health effects and financial costs of strategies to reduce salt intake and control tobacco use. Lancet. 2007; 370 (9604): 2044-53.

8. World Health Organization. Guideline. Sodium intake for adults and children. Geneva, World Health Organization (WHO), 2012. Disponible en: https://www. ncbi.nlm.nih.gov/books/NBK133309/pdf/Bookshelf_ NBK133309.pdf. [Consultado el 1 de Julio de 2020].

9. O’Donnell M, Mente A, Rangarajan S, McQueen MJ, Wang X, Liu L, et al. Urinary sodium and potassium excretion, mortality, and cardiovascular events. N Engl J Med. 2014; 371 (7): 612-23.

10. Mente A, O’Donnell M, Rangarajan S, Dagenais G, Lear S, McQueen M, et al. Associations of urinary sodium excretion with cardiovascular events in individuals with and without hypertension: a pooled analysis of data from four studies. Lancet. 2016; 388 (10043): 465-75.

11. Cogswell ME, Loria CM, Terry AL, Zhao L, Wang CY, Chen TC, et al. Estimated 24-Hour Urinary Sodium and Potassium Excretion in US Adults. JAMA. 2018; 319 (12): 1209-20.

12. McLean R. Measuring population sodium intake: a review of methods. Nutrients. 2014; 6 (11): 4651-62.

13. McLean R, Cameron C, Butcher E, Cook NR, Woodward M, Campbell NR. Comparison of 24-hour urine and 24-hour diet recall for estimating dietary sodium intake in populations: A systematic review and meta-analysis. J Clin Hypertens (Greenwich). 2019; 21 (12): 1753-62.

14. Campino C, Hill C, Baudrand R, Martínez-Aguayo A, Aglony M, Carrasco CA. Usefulness and Pitfalls in Sodium Intake Estimation: Comparison of Dietary Assessment and Urinary Excretion in Chilean Children and Adults. Am J Hypertens. 2016; 29 (10): 1212-7.

15. John KA, Cogswell ME, Campbell NR, Nowson CA, Legetic B, Hennis AJ, et al. Accuracy and Usefulness of Select Methods for Assessing Complete Collection of 24-Hour Urine: A Systematic Review. J Clin Hypertens (Greenwich). 2016; 18 (5): 456-67.

16. Tanaka T, Okamura T, Miura K, Kadowaki T, Ueshima $\mathrm{H}$, Nakagawa $\mathrm{H}$, et al. A simple method to estimate populational 24-h urinary sodium and potassium excretion using a casual urine specimen. J Hum Hypertens. 2002; 16 (2): 97-103.

17. Kawasaki T, Itoh K, Uezono K, Sasaki H. A simple method for estimating $24 \mathrm{~h}$ urinary sodium and potassium excretion from second morning voiding urine specimen in adults. Clin Exp Pharmacol Physiol. 1993; 20 (1): 7-14.

18. Toft U, Cerqueira C, Andreasen AH, Thuesen BH, Laurberg P, Ovesen L, et al. Estimating salt intake in a Caucasian population: can spot urine substitute 24-hour urine samples? Eur J Prev Cardiol. 2014; 21 (10): 1300-7.

19. Brown IJ, Dyer AR, Chan Q, Cogswell ME, Ueshima $\mathrm{H}$, Stamler J, et al. Estimating 24-hour urinary sodium excretion from casual urinary sodium concentrations in Western populations: the INTERSALT study. Am J Epidemiol. 2013; 177 (11): 1180-92.

20. Nerbass FB, Pecoits-Filho R, McIntyre NJ, McIntyre CW, Taal MW. Development of a formula for estimation of sodium intake from spot urine in people with chronic kidney disease. Nephron Clin Pract. 2014; 128 (1-2): 61-6.

21. Rhee MY, Kim JH, Shin SJ, Gu N, Nah DY, Hong KS, et al. Estimation of 24-hour urinary sodium excretion using spot urine samples. Nutrients. 2014; 6 (6): 2360-75.

22. Allen NB, Zhao L, Loria CM, Van Horn L, Wang CY, 
Pfeiffer CM, et al. The Validity of Predictive Equations to Estimate 24-Hour Sodium Excretion: The MESA and CARDIA Urinary Sodium Study. Am J Epidemiol. 2017; 186 (2): 149-59.

23. Price CP, Newall RG, Boyd JC. Use of protein:creatinine ratio measurements on random urine samples for prediction of significant proteinuria: a systematic review. Clin Chem. 2005; 51 (9): 1577-86.

24. Nowson CA, Lim K, Campbell NR, O’Connell SL, He FJ,
Daly RM. Impact of fractional excretion of sodium on a single morning void urine collection as an estimate of 24-hour urine sodium. J Clin Hypertens (Greenwich). 2019; 21 (12): 1763-70.

25. Jędrusik P, Symonides B, Gaciong Z. Performance of 24-hour urinary creatinine excretion-estimating equations in relation to measured 24-hour urinary creatinine excretion in hospitalized hypertensive patients. Sci Rep. 2019; 9 (1): 3593. 